\title{
A Hanf number for saturation and omission
}

\author{
John T. Baldwin* \\ University of Illinois at Chicago \\ Saharon Shelah \\ Hebrew University of Jerusalem \\ Rutgers University
}

January 4, 2011

\begin{abstract}
Suppose $\boldsymbol{t}=\left(T, T_{1}, p\right)$ is a triple of two countable theories $T \subseteq T_{1}$ in vocabularies $\tau \subset \tau_{1}$ and a $\tau_{1}$-type $p$ over the empty set. We show the Hanf number for the property: There is a model $M_{1}$ of $T_{1}$ which omits $p$, but $M_{1} \uparrow \tau$ is saturated is essentially equal to the Löwenheim number of second order logic. In Section 4 we make exact computations of these Hanf numbers and note some interesting distinctions between 'first order' and 'second order quantification'. In particular, we show that if $\kappa$ is uncountable, $h^{3}\left(L_{\omega, \omega}(Q), \kappa\right)=h^{3}\left(L_{\omega_{1}, \omega}, \kappa\right)$, where $h_{3}$ is the 'normal' notion of Hanf function (Definition 4.13.)
\end{abstract}

Newelski asked in [New] whether it is possible to calculate the Hanf number of the following property $P_{N}$. In a sense made precise in Theorem 0.2 , we show the answer is no. In accordance with the original question, we focus on countable vocabularies for the first three sections. We deal with extensions to larger vocabularies in Section 4.

Definition 0.1 We say $M_{1} \models \boldsymbol{t}$ where $\boldsymbol{t}=\left(T, T_{1}, p\right)$ is a triple of two theories in vocabularies $\tau \subset \tau_{1}$, respectively, $T \subseteq T_{1}$ and $p$ is a $\tau_{1}$-type over the empty set if $M_{1}$ is a model of $T_{1}$ which omits $p$, but $\bar{M}_{1} \uparrow \tau$ is saturated. Let $\boldsymbol{K}_{\boldsymbol{t}}$ denote the class of models $M_{1}$ which satisfy $\boldsymbol{t}$.

For $\boldsymbol{K}=\boldsymbol{K}_{\boldsymbol{t}}$, with $\boldsymbol{t}$ in a countable vocabulary, let $P_{N}^{c}\left(\boldsymbol{K}_{\boldsymbol{t}}, \lambda\right)$ hold if $\left|\tau_{1}\right| \leq \aleph_{0}$ and for some $M_{1}$ with $\left|M_{1}\right|=\lambda, M_{1} \models \boldsymbol{t} . P_{N}^{f}\left(\boldsymbol{K}_{\boldsymbol{t}}, \lambda\right)$ is the same property restricted to triples where $T_{1}$ and $T$ are finitely axiomatizable in finite vocabularies and $p$ is definable in second order logic.

Recall Hanf's observation [Han60] that for any such property $P(\boldsymbol{K}, \lambda)$, where $\boldsymbol{K}$ ranges over a set of classes of models, there is a cardinal $\kappa=H(P)$ such that $\kappa$ is the least cardinal satisfying: if $P(\boldsymbol{K}, \lambda)$ holds for some $\lambda \geq \kappa$ then $P(\boldsymbol{K}, \lambda)$ holds for

${ }^{*}$ We give special thanks to the Mittag-Leffler Institute where this research was conducted. This is paper 958 in Shelah's bibliography. Baldwin was partially supported by NSF-0500841. Shelah thanks the Binational Science Foundation for partial support of this research. 
arbitrarily large $\lambda . H(P)$ is called the Hanf number of $P$. E.g. $P(\boldsymbol{K}, \lambda)$ might be the property that $\boldsymbol{K}$ has a model of power $\lambda$. Similarly the Löwenheim number $\ell(P)$ of a set $P$ of classes is the least cardinal $\mu$ such that any class $\boldsymbol{K} \in P$ that has a model has one of cardinality $\leq \mu$.

Theorem 0.2 Assume the collection of $\lambda$ with $\lambda^{<\lambda}=\lambda$ is a proper class. $H\left(P_{N}^{f}\right)=$ $\ell\left(L^{I I}\right)$ where $L^{I I}$ denotes the collection of sentences of second order logic.

Since $H\left(P_{N}^{c}\right) \geq H\left(P_{N}^{f}\right)$, this shows that the Hanf number in the abstract is at least $\ell\left(L^{I I}\right)$, as asserted. In Section 1 we introduce a variant $\ell^{2}\left(L^{I I}\right)$ on the Löwenheim number of second order logic which is 'essentially equal' to $\ell\left(L^{I I}\right)$ (i.e. equal modulo a mild set theoretic hypothesis: Assumption 0.3). It is fairly easy to show (Claim 2.9) $\ell^{2}\left(L^{I I}\right) \geq H\left(P_{N}^{f}\right)$ giving the 'essentially equal' of the abstract. We will replace this 'essential equality' with an exact computation and deal with uncountable languages in Section 4.

Jouko Vaananen provided the following summary of the effect of this result by indicating the size of $\ell\left(L^{I I}\right) . \ell\left(L^{I I}\right)$ is bigger than the first (second, third, etc) fixed point of any normal function on cardinals that itself can be described in second order logic. For example it is bigger than the first $\kappa$ such that $\kappa=\beth_{\kappa}$, bigger than the first $\kappa$ such that there are $\kappa$ cardinals $\lambda$ below $\kappa$ such that $\lambda=\beth_{\lambda}$, etc. It is easy to see that if there are measurable (inaccessible, Mahlo, weakly compact, Ramsey, huge) cardinals, then the Lowenheim number of second order logic exceeds the first of them (respectively, the first inaccessible, Mahlo, weakly compact, Ramsey, huge) (and second, third, etc). So even under $V=L$, the Löwenheim number is bigger than any 'large' cardinal that is second order definable and consistent with $V=L$. Such results are discussed in Vaananen's paper "Hanf numbers of unbounded logics"[Vaa79]. A result of Magidor [Mag71] shows the Lowenheim number of second order logic is always below the first supercompact. Vaananen's paper "Abstract logic and set theory II: Large cardinals" gives lower bounds for the Lowenheim number of equicardinality quantifiers and thus a fortiori for second order logic [Vaa82]. In simple terms, if $E(\kappa)$ is the statement that $2^{\kappa} \geq \kappa^{++}$then the first $\kappa$ cardinals (if any) such that $E(\kappa)$ holds is less than the Lowenheim number of second order logic. This shows that by forcing we can push the Lowenheim number up at will.

We make the following assumption throughout.

Assumption 0.3 Assume the collection of $\lambda$ with $\lambda^{<\lambda}=\lambda$ is a proper class.

This assumption follows from GCH, but if GCH fails badly the only such cardinals are strongly inaccessible. The key point for our use of the condition is that $\lambda^{<\lambda}=$ $\lambda>\left|\tau_{T}\right|+\aleph_{0}$ is a sufficient condition for the existence of a saturated model in $\lambda$ of a complete theory $T$; if $T$ is unstable $\lambda^{<\lambda}=\lambda$ is also necessary. We will explore this issue for stable theories, in the absence of Assumption 0.3, elsewhere. In Section 1 we review some properties of second order logic and show the equality of two 'Löwenheim numbers' in our context. In Section 2, we state two technical results, prove one, and deduce Theorem 0.2 from them. In Section 3, we prove the more difficult technical result. In Section 4, we code syntax more carefully and obtain a uniform equivalence for vocabularies of all cardinalities. 
Newelski's question arose in the study of the model theory of groups and the existence of groups with bounded orbits. The authors acknowledge very fruitful discussions with Jouko Väänänen and Tapani Hyttinen concerning the material of this paper. We also thank the referee for an unusually detailed and helpful series of reports.

\section{Some Second Order Logic}

By (pure) second order logic, $L^{I I}$, we mean the logic with individual variables and variables for relations of all arities but no non-logical constants. The atomic formulas are equalities between variables and expressions $X(\mathbf{x})$ where $X$ is an $n$-ary relation variable and $\mathrm{x}$ is an $n$-tuple of individual variables. Note that a structure $A$ for this logic is simply a set so is determined entirely by its cardinality. But we use the full semantics; the $n$-ary relation variables range over all $n$-ary relations on $A$.

We explain the connection of our restriction to $\lambda$ with $\lambda=\lambda^{<\lambda}$ to the computation of some variants on the Hanf and Löwenheim numbers.. In general for any class $\boldsymbol{K}$ of models write $\operatorname{spec}(\boldsymbol{K})$ for the collection of $\lambda$ such that there is a model in $\boldsymbol{K}$ with cardinality $\lambda$.

Definition 1.1 Let $\psi$ be a sentence of second order logic.

1. $\operatorname{spec}^{1}(\psi)=\{\lambda: \lambda \mid \psi \psi\}$.

2. $\operatorname{spec}^{2}(\psi)=\left\{\lambda: \lambda=\lambda^{<\lambda} \wedge \lambda \models \psi\right\}$.

Note that there is a sentence $\chi$ in second order logic which has a model of size $\lambda$ if and only if $\lambda^{<\lambda}=\lambda$. Namely, let $\chi$ assert there is an extensional relation $R$ on sets such that each element denotes, via $R$, a set of smaller cardinality than the universe and each such set is coded by $R$. We will generally write $\lambda^{<\lambda}=\lambda$ to denote this sentence.

Definition 1.2 Define $H^{2}$ and $\ell^{2}$ to be the Hanf and Lowenheim numbers with respect to $\operatorname{spec}^{2}$.

We'll write $\ell^{1}$ for $\ell$ and $H^{1}$ for $H$ where it is convenient for comparison. Note the following easy transformations in second order logic.

Fact 1.3 Fix $\phi \in L^{I I}$.

1. $\operatorname{spec}^{1}(\phi)$ :

(a) There is a $\phi_{1} \in L^{I I}$ with $\min (\operatorname{spec}(\phi))<\min \left(\operatorname{spec}\left(\phi_{1}\right)\right)$.

(b) If $\operatorname{spec}(\phi)$ is bounded and nonempty there is a $\phi_{2} \in L^{I I}$ with $\operatorname{spec}\left(\phi_{2}\right)$ bounded and nonempty and $\sup (\operatorname{spec}(\phi))<\sup \left(\operatorname{spec}\left(\phi_{2}\right)\right)$.

2. $\operatorname{spec}^{2}(\phi)$ :

(a) There is a $\phi_{3} \in L^{I I}$ with $\min (\operatorname{spec}(\phi))<\min \left(\operatorname{spec}\left(\phi_{3}\right)\right)$ and if $\lambda \models \phi_{3}$, $\lambda^{<\lambda}=\lambda$. 
(b) If $\operatorname{spec}^{2}(\phi)$ is bounded and nonempty there is a $\phi_{4} \in L^{I I}$ with $\operatorname{spec}^{2}\left(\phi_{4}\right)$ bounded and nonempty and $\sup \left(\operatorname{spec}^{2}\left(\phi_{4}\right)\right)<\sup \left(\operatorname{spec}^{2}\left(\phi_{4}\right)\right)$.

These transformations imply:

Fact 1.4 1. $H^{1}\left(L^{I I}\right), H^{2}\left(L^{I I}\right), \ell^{1}\left(L^{I I}\right), \ell^{2}\left(L^{I I}\right)$ are strong limit cardinals.

2. There is no sentence attaining any of these values exactly. (E.g., there is no $\phi \in L^{I I}$ with $\sup (\operatorname{spec}(\phi))=H^{1}\left(L^{I I}\right)$.)

3. For either spectrum, $\ell^{i}\left(L^{I I}\right)=\sup \left\{\min \left\{\operatorname{spec}^{i}(\phi)\right\}: \phi \in L^{I I}\right.$ has a model $\}$ and similarly $H^{i}\left(L^{I I}\right)=\sup \left\{\sup \left\{\operatorname{spec}^{i}(\phi)\right\}: \phi \in L^{I I}\right.$ is bounded $\}$.

Note that any logic satisfying Fact 1.3 will also satisfy Fact 1.4. We use this observation without comment in studying infinitary second order logics in Section 4.

Using Assumption 0.3 we can show:

Lemma 1.5 $H\left(L^{I I}\right)=H^{2}\left(L^{I I}\right), \ell\left(L^{I I}\right)=\ell^{2}\left(L^{I I}\right)$

Proof. One direction is easy. For every sentence $\psi$ of second order logic, there is a sentence $\psi^{*}$ such that:

$$
\operatorname{spec}^{2}(\psi)=\operatorname{spec}^{1}\left(\psi^{*}\right) .
$$

$\psi^{*}$ just expresses the conjunction of $\psi$ with $\lambda^{<\lambda}=\lambda$. Recall Fact 1.4.3 Since every 2 -spectrum is a 1 -spectrum $\ell^{2}\left(L^{I I}\right) \leq \ell^{1}\left(L^{I I}\right)$ and $H^{2}\left(L^{I I}\right) \leq H^{1}\left(L^{I I}\right)$.

But the opposite inequality also holds. Let $\phi$ be a sentence with a non-empty 2spectrum. Let $f(\lambda)$ denote the least $\mu>\lambda$ with $\mu^{<\mu}=\mu$. It is easy to construct for each second order sentence $\phi$ a sentence $\phi^{*}$ such that

$$
\operatorname{spec}\left(\phi^{*}\right)=\operatorname{spec}^{2}\left(\phi^{*}\right)=\{f(\lambda): \lambda \in \operatorname{spec}(\phi)\} .
$$

Clearly the map $\phi \mapsto \phi^{*}$ shows $\ell^{2}\left(L^{I I}\right) \geq \ell^{1}\left(L^{I I}\right)$ and $H^{2}\left(L^{I I}\right) \geq H^{1}\left(L^{I I}\right)$.

\section{The main result}

Recall our notation from Definition 0.1.

Notation 2.1 We will write $\boldsymbol{t}$ (possibly with subscripts) for a triple $\left(T, T_{1}, p\right)$. The expression ' $\boldsymbol{t}$ has a model in $\lambda$ ' means there is a model of $T_{1}$ with cardinality $\lambda$ that omits $p$ and whose reduct to $L(T)=\tau$ is saturated.

Convention 2.2 When $\tau_{1}$ is finite we consider it to be a subset of $\omega$. Thus the set of first order $\tau_{1}$-sentences is recursive and we can code them as natural numbers.

We concentrate first on $P_{N}^{f}\left(\boldsymbol{K}_{\boldsymbol{t}}, \lambda\right)$ from Definition 0.1. We need some additional coding to handle non-finitely axiomatizable theories and consider this generalization in Section 4. We begin by clarifying a notion from Definition 0.1. 
Definition 2.3 A type $p$ in a vocabulary satisfying Convention 2.2 is definable in second order logic if we can code the type as a subset $A_{p}$ of $\omega$ so that in the vocabulary with constant symbol 0 and relation symbol $S$ there is a second order sentence $\psi$ and second order formula $\varphi(x)$ satisfying the following condition. For the first cardinal $\lambda$ which satisfies $\psi$ if $M$ is $(\lambda, 0, S)$, with 0 interpreted as 0 and $S$ as successor on the natural numbers, then $A_{p}=\{n: M \models \varphi(n)\}$.

Now for convenience we restrict our triples to those satisfying the convention. Formally:

Notation 2.4 $\mathcal{T}^{f}$ denotes the set of triples $\boldsymbol{t}$ as in Definition 2.1 such that $\tau_{1}$ satisfies Convention 2.2, $T$ is finitely axiomatizable and $p$ is second order definable.

Theorem 2.5 For every second order sentence $\phi$, there is a triple $\boldsymbol{t}_{\phi} \in \mathcal{T}^{f}$ such that if $\lambda^{<\lambda}=\lambda$, then the following are equivalent:

1. $\boldsymbol{t}_{\phi}$ has a model in $\lambda$.

2. $\phi$ has a model in every cardinal strictly less than $\lambda$.

We prove Theorem 2.5 in Section 3.

Lemma 2.6 For every $\boldsymbol{t} \in \mathcal{T}^{f}$ there is a second order $\phi_{\boldsymbol{t}}$, such that $\phi_{\boldsymbol{t}}$ has a model in $\lambda$ if and only if $\boldsymbol{t}$ has a model in $\lambda$.

Proof. Recalling the restrictions involved in $\mathcal{T}^{f}$, it is easy to write a second order sentence $\theta$ such that $M \models \theta$ if and only if $M \models T_{1}, M$ omits $p$ and $M\lceil\tau$ is saturated. $\square_{2.6}$

We could strengthen Lemma 2.6 by restricting the second order quantification to sets of size strictly less than the size of the model, but that is not important here. We now deduce Theorem 0.2 from Theorem 2.5 and Lemma 2.6. We use the following notation.

Notation 2.7 Spec $(\boldsymbol{t})$ is the collection of cardinals $\lambda$ such that there is an $M_{1}$ satisfying $\boldsymbol{t}$ with $\left|M_{1}\right|=\lambda$.

We have not established that the Hanf and Lowenheim numbers for the $P_{N}$ satisfy Fact 1.4. This complicates the argument for the following two results.

Claim 2.8 $H\left(P_{N}^{f}\right) \leq \ell^{2}\left(L^{I I}\right)$ where $L^{I I}$ denotes second order logic.

Proof. Lemma 2.6 shows that for any $\boldsymbol{t} \in \mathcal{T}^{f}$, there is a $\phi_{\boldsymbol{t}} \in L^{I I}$ with $\operatorname{spec}(\boldsymbol{t})=$ $\operatorname{spec}\left(\phi_{\boldsymbol{t}}\right)$. Suppose for contradiction that $H\left(P_{N}^{f}\right)>\ell^{2}\left(L^{I I}\right)$. Then there is a triple $\mathbf{t} \in \mathcal{T}^{f}$ such that $\sup (\operatorname{spec}(\mathbf{t})) \geq \ell^{2}\left(L^{I I}\right)$.

Let $\mathbf{C}=\left\{\mu: \mu=\mu^{<\mu}\right\}$. Choose $\psi_{\mathbf{t}} \in L^{I I}$ so that $\mu \models \psi_{\mathbf{t}}$ iff for every infinite cardinal $\kappa \in \mathbf{C} \cap \mu$ there is $\theta \in[\kappa, \mu) \cap \operatorname{spec}(\mathbf{t})$.

Let $\lambda_{\mathbf{t}}$ be the minimal element of $\mathbf{C} \cap \operatorname{spec}(\mathbf{t})^{c}$. Then $\lambda_{\mathbf{t}} \geq \ell^{2}\left(L^{I I}\right)$. For any $\boldsymbol{t}$, the definition of $P_{N}^{f}$ guarantees that if $\lambda^{<\lambda}=\lambda, \mu>\lambda$ and some $M \models t$ has cardinality 
$\mu$ then some $N=t$ has cardinality $\lambda$ (take a saturated elementary submodel). Thus, $\mu=\psi_{\mathbf{t}}$ if and only if every $\kappa \in \mathbf{C} \cap \mu$ belongs to $\operatorname{spec}(\mathbf{t})$.

Now $\operatorname{spec}\left(\psi_{\mathbf{t}}\right)$ is exactly $\left\{\mu: \mu \leq \lambda_{\mathbf{t}}\right\}$, whence $\operatorname{spec}\left(\neg \psi_{\mathbf{t}}\right)$ is $\left\{\mu: \mu>\lambda_{\mathbf{t}}\right\}$. So the Löwenheim number of $\neg \psi_{\mathbf{t}}$ is $\left(\lambda_{\mathbf{t}}\right)^{+}>\ell^{2}\left(L^{I I}\right)$ and this contradiction completes the proof.

Lemma 2.9 $H\left(P_{N}^{f}\right) \geq \ell^{2}\left(L^{I I}\right)$ where $L^{I I}$ denotes second order logic.

Proof. Suppose for contradiction that there is a second order sentence $\psi$ such that $\lambda_{0}=\min \left(\operatorname{spec}^{2}(\psi)\right) \geq H\left(P_{N}^{f}\right)$. By the definition of $\operatorname{spec}^{2}, \lambda_{0}^{<\lambda_{0}}=\lambda_{0}$. Let $\hat{\psi}$ express $(\exists U)\left(\psi^{U} \wedge|U|^{<|U|}=|U|\right)$. We apply Theorem 2.5 to $\neg(\hat{\psi})$. Note that $\hat{\psi}$ is true on all cardinals $\geq \lambda_{0}$ and false on all $\mu<\lambda_{0}$. By Theorem 2.5, $\lambda_{0} \models \boldsymbol{t}_{\neg(\hat{\psi})}$ and $\lambda_{0} \geq H\left(P_{N}^{f}\right)$. So $\boldsymbol{t}_{\neg(\hat{\psi})}$ and therefore $\neg(\hat{\psi})$ has arbitrarily large models. But $\neg(\hat{\psi})$ has no models larger than $\lambda_{0}$. This contradiction yields the theorem.

In the next section we prove the crucial Theorem 2.5. In the last section we remove the restrictions to finitely axiomatizable theories and countable languages.

\section{Essential Lemmas}

Now we prove Theorem 2.5. For convenience, we list here the two vocabularies. We describe the axioms of $T$ and $T_{1}$ below.

Notation 3.1 1. $\tau$ contains unary predicates $Q_{1}, Q_{2}$, a binary relation $R$ and partial binary functions $F$ and $F_{2}$. It contains two constant symbols $c_{0}, c_{\omega}$ and $a$ unary function symbol $g$.

2. $\tau_{1}$ adds a unary predicate $Q_{0}$ and a binary relation $<_{1}$.

Remark 3.2 (Proof Sketch) For each second order $\phi$, we construct a triple $\boldsymbol{t}_{\phi}$. But most of the construction is independent of the particular $\phi$ and so we first construct a theory $T_{1}$ which does not depend on $\phi$. The vocabulary $\tau$ will contain unary predicates $Q_{1}, Q_{2}$. The axioms will assert that $Q_{1}, Q_{2}$ partition the universe. $Q_{0}$ is in $\tau_{1}$. Omission of the type $p$ will guarantee that $Q_{0} \subset Q_{1}$ is countable. Omission of the type in a model $M$ of $T_{1}$ whose $\tau$-reduct is $\aleph_{1}$-saturated and some coding involving the partial order $<_{0}$ in $\tau$ will guarantee that $Q_{1}(M)$ is well-ordered by a relation symbol $<_{1}$ in $\tau_{1}$. A relation symbol $R$ in $\tau$ will code subsets of $Q_{1}$ by elements of $Q_{2}$. Thus first order quantification on $Q_{2}$ will encode second order quantification on $Q_{1}$. In particular, we can code a given second order sentence $\phi$ and thus extend $T_{1}$ to $T_{\phi}$. But the encoding guarantees 'correctness' only on subsets whose every subset is coded in $Q_{2}$. The construction will guarantee that if $\mu<\lambda$ and $M$ is $\lambda$-saturated, then $\mu$ is a $<_{1}$-initial segment $Q_{1}$. Since $\mu<\lambda$ each subset of $\mu$ is coded by a type of size $\mu$ so the encoded semantics is correct and $\mu$ is a model of $\phi$.

Beginning the Proof of Theorem 2.5. We gradually introduce the vocabulary and theory explaining the use of various predicates as they are introduced; we repeat a bit 
of the proof sketch. Below we say certain conditions hold to mean they hold in any model of $T$. We first describe $\tau$ and $T$. In particular, $\tau$ contains unary predicates $Q_{1}, Q_{2}$ that partition the universe.

There is a binary relation $<_{0}$, which is a partial order of $Q_{1}$. There is a partial function $F$ mapping $Q_{1} \times Q_{1}$ into $Q_{1}$. We write $F_{a}$ for the partial function from $Q_{1}$ into $Q_{1}$ indexed by $a$. Any model of $T$ satisfies: $a \leq_{0} b$ implies $F_{a} \subset F_{b}$.

We have two further properties of $F . F_{c_{0}}$ is the empty function. For every $a \in Q_{1}$ and every $e \in Q_{1}$, if $e \notin \operatorname{dom} F_{a}$, then there are $b, d \in Q_{1}$ with $a<_{0} b$ and $F_{b}=$ $F_{a} \cup\{\langle e, d\rangle\}$.

Further there is a pairing function $F_{2}$ on $Q_{1}$ and an extensional relation $R$ between $Q_{1}$ and $Q_{2}$ so that each element of $Q_{2}$ codes a subset of $Q_{1}$ via $R$. We write $U_{b}$ for $\{a: R(b, a)\}$ (for $a \in Q_{1}$ and $b \in Q_{2}$ ).

$T$ asserts that $Q_{1}$ is preserved by $g$, that $g$ is a permutation, and $Q_{1}\left(c_{0}\right)$.

The set of $\left\{U_{a}: a \in Q_{2}\right\}$ is closed under Boolean operations and if $U_{b}$ is such a set so is $F_{a}\left(U_{b}\right)$ for any $a \in Q_{1}$. For each $a \in Q_{1}$, there is $b \in Q_{2}$ such that $U_{b}=\left\{c: c<_{1} a\right\}$.

Secondly, we turn to the description of $\tau_{1}$ and $T_{1}$. In $\tau_{1}$, there is a binary $\tau_{1}$ relation $<_{1}$, which is a linear order of $Q_{1}$ and such that on $Q_{1}, x<_{1} g(x)$ and $x<_{1} c_{\omega}$ implies $g(x)<_{1} c_{\omega}$. Further there is a unary relation $Q_{0}$ such that $Q_{0} \subset Q_{1}$ and $T_{1}$ asserts $c_{0}, c_{\omega}$ are in $Q_{0}, Q_{0}$ is preserved by $g$ and if $x<_{1} c_{\omega}$ then $Q_{0}(x)$. Thus, each $g^{i}\left(c_{0}\right) \in Q_{0}$. Thus, $\left\{g^{i}\left(c_{0}\right): i<\omega\right\} \cup\left\{c_{\omega}\right\}$ name countably many elements of $Q_{1}$ which are $<_{1}$ - ordered in order type $\omega+1$. $T_{1}$ further asserts $\left(Q_{1},<_{1}\right)$ is 'internally well-ordered' in the following sense. For every $a \in Q_{2}$, if $U_{a}$ is non-empty, it has a $<$-least element.

The type $p$ asserts $Q_{0}(x)$ and $x$ is not a $g^{i}\left(c_{0}\right)$ for any $i<\omega$. Thus if $p$ is omitted in a model $M, Q_{0}(M)=\left\{g^{i}\left(c_{0}\right): i<\omega\right\} \cup\left\{c_{\omega}\right\}$.

Claim 3.3 If a model $M$ of $T_{1}$ is such that its reduct to $\tau$ is an $\aleph_{1}$-saturated model of $T$ but $M$ omits $p,\left(Q_{1},<_{1}\right)$ is a well-ordering in $M$.

Proof. Suppose there is a countable $<_{1}$-descending chain $B=\left\{b_{i}: i<\omega\right\}$ in $\left(Q_{1},<_{1}\right)$. Using the properties of $F$, we can define a $<_{0}$-increasing chain of $a_{n}$ in $Q_{1}$ such that $F_{a_{n}}=\left\{\left\langle c_{0}, b_{0}\right\rangle, \ldots\left\langle g^{n}\left(c_{0}\right), b_{n}\right\rangle\right\}$, where the $g^{i}\left(c_{0}\right)$ are images of $c_{0}$ by iterating $g$. Since the model is $\aleph_{1}$-saturated there is an $a_{\omega} \in Q_{1}$ such that each $F_{a_{n}} \subset F_{a_{\omega}}$. But then $B=F_{a_{\omega}}\left(\left\{g^{i}\left(c_{0}\right): i<\omega\right\}\right)$. Note that while the choice of $b_{i}$ involved the $\tau_{1}$-symbol $<_{1}$, the existence of $a_{\omega}$ is by the consistency of a $\tau$-type so the use of saturation is legitimate.

Since $M$ omits $p,\left\{g^{i}\left(c_{0}\right): i<\omega\right\}=Q_{0}(M)=\left\{a: a<_{1} c_{\omega}\right\}$ and therefore is coded by an element of $Q_{2}$. By the closure properties of the coded sets, $B=U_{d}$ for some $d \in Q_{2}$. This contradicts the internal well-ordering of $Q_{1}$.

Now translate $\phi$ to the first order formula $\phi^{*}(v)$ by translating each bound second order variable $\mathrm{X}$ to a first order formula in $x$ and $v$. Replace each occurrence of $X(z)$ by $R(z, v) \wedge R(z, x)$. This translation has the following consequence. (This is immediate for monadic second order but we included a pairing function $F_{2}$ on $Q_{1}$ so it extends to arbitrary sentences.) 
Fact 3.4 If $M \models T, a \in Q_{2}(M)$ and each subset of $U_{a}$ is coded by an element of $Q_{2}(M)$, then $M \models \phi^{*}(a)$ if and only $U_{a}(M) \models \phi$.

Add the following axiom to $T_{1}$ to obtain the theory $T_{\phi}$ :

$$
(\forall u)(\forall w)\left[\left((\forall z) R(z, w) \leftrightarrow z<_{1} u\right) \rightarrow \phi^{*}(w)\right] .
$$

This completes $\mathbf{t}_{\phi}$ as $\left\langle T, T_{\phi}, p\right\rangle$.

Claim 3.5 If $\mu<\lambda=\lambda^{<\lambda}$ and $M$ is model of $\phi$ with cardinality $\lambda$ that omits $p$ but whose reduct to $\tau$ is saturated then $\mu=\phi$.

Conversely, if $\phi$ is true on all $\mu<\lambda=\lambda^{<\lambda}$, there is a model $M_{1}$ of $T_{\phi}$ with cardinality $\lambda$ that omits $p$ but whose reduct to $\tau$ is saturated.

Proof. Since $\mu<\lambda, \mu$ is an initial segment of $Q_{1}$ so $\mu=\left\{a \in Q_{1}: R(y, d)\right\}$ for some $d \in Q_{2}$. But then each subset $Y$ of $\mu$ gives rise to a type:

$$
q_{Y}(x)=\{R(y, d)\} \cup\{R(y, x): y \in Y\} \cup\{\neg R(y, x): y \notin Y\} .
$$

For each $Y$ the $\tau$-type $q_{Y}(x)$ has cardinality less than $\lambda$ and so is realized by saturation. We finish by Fact 3.4.

For the converse, well-order $Q_{1}$ by $<_{1}$ in order type $\lambda$. Add in $Q_{2}$ a code for each subset of cardinality $<\lambda$. Let the $F_{a}$ list the partial functions of cardinality less than $\lambda$ from $Q_{1}$ to $Q_{1}$ and let $<_{0}$ denote the natural partial ordering on $Q_{1}$ induced by inclusion of the named functions. Since $\phi$ is true below $\lambda$, each infinite initial segment in $\lambda$ defines a model of $\phi$ and the definition of $T_{\phi}$ shows that we have a saturated model of $T$ when we take the reduct to $\tau$. Finally, let $Q_{0}$ include exactly the first $\omega$ elements of $Q_{1}$.

\section{Concluding the Proof of Theorem 2.5.}

Letting $\boldsymbol{t}_{\phi}$ be the triple $\left(T, T_{\phi}, p\right)$ we have a triple satisfying Theorem 2.5. $\quad \square_{2.5}$

\section{The exact strength}

In this section we remove the restrictions to finitely axiomatizable theories and countable languages. In Theorem 4.12 we prove actual equality of the Hanf number studied here (for any triple of theories and types of any cardinality) with a Löwenheim number of second order logic; the cost is that we must move into infinitary second order logic and (in the proof) allow relation constants (i.e. predicate symbols other than equality) in the vocabulary of the second order sentence.

Instead of Theorem 2.5 we could slightly more easily prove

$$
H\left(P_{N}^{f}\right) \leq \ell^{2}\left(L^{I I}\right) \leq H\left(P_{N}^{c}\right),
$$

which gives our answer to Newelski's question but is not quite as sharp. That is, if we had just required $\boldsymbol{t}_{\phi}$ in Theorem 2.5 to be in a countable language rather than finitely axiomatizable, this would have no effect on the proof of Lemma 2.9 and it would have 
simplified the proof of Theorem 2.5 since we could have worked with countably many constants and omitted the function $g$. Similarly the arguments of Sections 2 and 3 extend from finitely axiomatizable to 'arithmetic' by coding a model of arithmetic in the second order sentence. And it is easy to see that the theory constructed in Theorem 2.5 is recursive. This observation is generalized in Theorem 4.12 to remove the restrictions on axiomatizability. The key idea is to see that we can use the same ideas as in Section 3 to code the syntax of infinitary second order logic by a triple $t$.

We extend our notion of second order logic in two ways. First we allow infinite conjunctions and strings of quantifiers. Secondly we now allow some relation constants instead of dealing with 'pure' second order logic.

Definition 4.1 1. $L_{\theta}$ denotes the $\theta$ stage in the construction of the inner model $L$.

2. Let $L_{\theta^{+}, \kappa}(I I)$ denote second order logic allowing strings of second order quantifiers of cardinality $<\kappa \leq \theta$ and conjunctions and disjunctions of cardinality $\leq \theta$.

Remark 4.2 Again using Assumption 0.3, note that as in Fact 1.3 the Löwenheim number of $L_{\theta^{+}, \kappa}(I I)$ is a strong limit cardinal of cofinality $>\theta$ and is an accumulation point of $\left\{\mu: \mu=\mu^{<\mu}\right\}$.

Notation 4.3 We denote by $L\left(I I, \tau_{*}\right)$ second order logic in the vocabulary $\tau_{*}$ consisting of constant symbols $c_{\kappa}, c_{\theta}, c_{\tau}, c_{\phi}$, a unary predicate $Q$, and a binary relation $R_{1}$.

Notation 4.4 Let $\boldsymbol{K}=\boldsymbol{K}_{\boldsymbol{t}}$, where the set of triples $\boldsymbol{t}$ is as in Notation 2.1 but requiring that $\tau_{1} \subset L_{\theta}$ and omitting the requirement that $p$ is second order definable. Then, $P_{N}^{\kappa}\left(\boldsymbol{K}_{\boldsymbol{t}}, \lambda\right)$ holds if $\left|\tau_{1}\right| \leq \kappa$ and for some $M_{1}$ with $\left|M_{1}\right|=\lambda, M_{1} \models \boldsymbol{t}$.

In the following $H(\mu)$ denotes the set of all sets whose transitive closure has cardinality less than $\mu$. We use implicitly that $\mu^{<\mu}=\mu$ implies that $\mu$ is regular and so $(H(\mu), \epsilon)$ satisfies all axioms of ZFC except power set.

We now construct a sentence $\psi \in L\left(I I, \tau_{*}\right)$ and for every $\tau \subset L_{\theta}$ and every $\phi$ in $L_{\theta^{+}, \kappa}(I I)(\tau)$ a set $A_{\tau, \phi}$ of ordinals so that $\psi$ and $A_{\tau, \phi} \operatorname{codes} \phi$.

Definition 4.5 Let $X$ be a transitive subset of $H\left(\theta^{+}\right)$with cardinality $\theta$. We say that $X$ is coded by $A \subseteq \theta$ if there is an injection $f$ from $X$ into $\theta$ such that:

$$
A=\{\operatorname{pr}(f(a), f(b)): a \in b \in X\} .
$$

Here pr is the standard pairing function on ordinals.

Now we show that the coding of $X$ by $A$ does not depend on the choice of $f$.

Lemma 4.6 If $A \subseteq \theta$ codes $X_{1}$ by $f_{1}$ and $X_{2}$ by $f_{2}$ then $X_{1}=X_{2}$.

Proof. We first note that for any $X, A$ if $A$ codes $X$ by $f$ then $f$ is a bijection between $X$ and $B=\{\alpha: \operatorname{pr}(\alpha, \beta) \in A \vee \operatorname{pr}(\beta, \alpha) \in A\}$. Applying this remark to 
$f_{1}, f_{2}$, we see $g=f_{2}^{-1} f_{1}$ is a bijection from $X_{1}$ onto $X_{2}$. But then $g$ is an isomorphism with respect to $\epsilon$ as it is easy to check (from the definition of coding) that for any $y_{1}, z_{1} \in X_{1}$ with $y_{2}=g\left(y_{1}\right)$ and $z_{2}=g\left(z_{1}\right), y_{1} \in z_{1}$ if and only if $y_{2} \in z_{2}$. That is, $y_{1} \in z_{1}$ if and only $\operatorname{pr}\left(f_{1}\left(y_{1}\right), f_{1}\left(z_{1}\right)\right) \in A$ if and only $\operatorname{pr}\left(f_{2}\left(y_{2}\right), f_{2}\left(z_{2}\right)\right) \in A$ if and only $y_{2} \in z_{2}$. But then since $\emptyset \in X_{1} \cap X_{2}, \epsilon$-induction yields that $X_{1}=X_{2} . \quad \square_{4.6}$

Lemma 4.7 If $\tau \subseteq L_{\theta}$ then each $\phi \in L_{\theta^{+},{ }_{\kappa}}(I I)(\tau)$ has at most $\theta$ subformulas, each in $\operatorname{tc}(\phi)$, and $\operatorname{tc}(\phi) \cup\{\phi\}$ is coded (in the sense of Definition 4.5) by a set $A_{\tau, \phi} \subset \theta$.

Proof. The standard construction of $\phi \in L_{\theta^{+}, \kappa}(I I)(\tau)$ yields that each formula is in $H\left(\theta^{+}\right)$, each subformula of $\phi \in \operatorname{tc}(\phi)$, and $\phi$ has at most $\theta$ subformulas. Define $A_{\tau, \phi}$ by Definition 4.5.

We first define certain set of ordinals $A_{\tau, \phi} \subseteq \theta$ in $V$ that codes $\tau$ and $\phi \in$ $L_{\theta^{+}, \kappa}(I I)(\tau)$. Here $\operatorname{tc}(X)$ denotes the transitive closure of $X$.

Definition 4.8 For every vocabulary $\tau \subseteq L_{\theta}$ and every sentence $\phi \in L_{\theta^{+},{ }_{\kappa}}(I I)(\tau)$ we define $A_{\tau, \phi} \subseteq \theta$ to be the set which codes $\left.\operatorname{tc}(\{\phi, \tau\}) \cup\{\phi, \tau\}\right)$ in the sense of Definition 4.5 .

Now we define the sentence $\psi \in L\left(I I, \tau_{*}\right)$, which does not depend on $\phi$ or $\tau$, so that the interpretation of a predicate $Q$ as $A_{\tau, \phi}$ in a model $M$ of $\psi$ will identify $\phi$ as the sentence under consideration. The function $G$ in Definition 4.9.2c simply formalizes the normal definition of truth.

Definition 4.9 We define a sentence $\psi \in L\left(I I, \tau_{*}\right) M \models \psi_{\phi}$ if and only if $M$ satisfies:

1. $\left(M, R_{1}^{M}\right) \approx(H(\mu), \epsilon)$ for some $\mu$ with $\mu^{<\mu}=\mu$.

2. the following $\tau^{*}$-axioms.

(a) $c_{\kappa}$ and $c_{\theta}$ are cardinals in the sense of $M$.

(b) $c_{\tau}$ is a vocabulary of cardinality $c_{\theta}$ contained in $L_{\theta}$.

(c) The set of formulas of $L_{c_{\theta}^{+}, c_{\kappa}}(I I)\left(c_{\tau}\right)$ are given their usual inductive definition in $M$ by a formula $\mu\left(x, c_{\theta}, c_{\kappa}, c_{\tau}\right) \in L(I I)\left(\tau_{*}\right)$.

(d) $c_{\phi} \in L_{c_{\theta}^{+}, c_{\kappa}}(I I)\left(c_{\tau}\right)$ (i.e. $M=\mu\left(c_{\phi}, c_{\theta}, c_{\kappa}, c_{\tau}\right)$ ).

(e) $Q(x) \rightarrow x R_{1} c_{\theta}$.

(f) $Q$ is the set of codes (in the sense of $M$ ) of $\operatorname{tc}(\{\phi, \tau\}) \cup\{\phi, \tau\})$ (where tc is with respect to $R_{1}$ ).

3. There is a definable function $G$ which defines truth on subsets $b$ of $M$ of sentences of $L_{\theta^{+},{ }_{\kappa}}\left(I I, c_{\tau}\right)$ : for every sentence $\chi, M \models G\left(b,{ }^{\prime} \chi^{\prime}\right)=1$ if and only if

$$
\left(M \uparrow\left\{a: a R_{1}^{M} b\right\}\right) \models \chi .
$$

(Here $\chi$ is actual sentence and ' $\chi$ ' is the member of $M$ mapped to $\chi$ under the isomorphism in Definition 4.9.1.) 
4. $G^{M}\left(b, c_{\phi}\right)=0$ if $M \models|b|<|M|$.

The goal of the following lemma is to compute the Löwenheim number of $L_{\theta^{+}, \kappa}(I I)$. Since it is certainly greater than $\theta$, we may assume $\lambda^{<\lambda}>\theta$.

Lemma 4.10 Fix $\kappa \leq \theta^{+}$, a vocabulary $\tau \subseteq L_{\theta}$ and $\phi \in L_{\theta^{+},{ }_{\kappa}}(I I)(\tau)$. Choose $A_{\tau, \phi}$ and $\psi \in L\left(I I, \tau_{*}\right)$ satisfying Definitions 4.8 and 4.9 , respectively. For any cardinal $\lambda=\lambda^{<\lambda}>\theta$, the following are equivalent.

1. $\phi$ has no model of cardinality $<\lambda$.

2. There is a model $\left(M, R_{1}^{M}, Q^{M}, c_{\theta}^{M}, c_{\kappa}^{M}, c_{\phi}^{M}, c_{\tau}^{M}\right)$ with cardinality $\lambda$ of the sentence $\psi$ defined in Definition 4.9 such that letting $P^{M}$ denote $(\{b: M \models$ ' $b$ is an ordinal $\wedge b R_{1}^{M} c_{\theta}$ \} and $P_{1}^{M}$ denote

$$
\left(\left\{b: M \models ' b \text { is an ordinal } \wedge b R_{1}^{M} c_{\kappa}\right\}:\right.
$$

(a) $\left(P^{M}, R_{1}^{M}\right)$ has order type $\theta$,

(b) $\left(P_{1}^{M}, R_{1}^{M}\right)$ has order type $\kappa$,

(c)

$$
A_{\tau, \phi}=\left\{\alpha<\theta: \text { for some } a \in Q^{M}, \alpha=\operatorname{otp}\left(\left\{b R_{1} c_{\theta}: b R_{1}^{M} a\right\}, R_{1}^{M}\right)\right\} .
$$

Proof. Suppose 2). Without loss of generality, we identify $\left(M, R_{1}^{M}\right)$ with $H(\lambda, \epsilon)$ yielding the equality in c). $\left(\tau \in H(\lambda)\right.$ since $\tau \subset L_{\theta}$.) Then $|M|=\lambda$, $\left(P^{M}, R_{1}^{M}\right)$ has order type $\theta$, and $A_{\tau, \phi}$ is the image of $Q^{M}$ under an isomorphism from $\left(P^{M}, R_{1}^{M}\right)$ to $\theta$. By the choice of $A_{\tau, \phi}$, the model $M$ correctly recognizes the vocabulary $\tau$ and the formula $\phi$. The function $G^{M}$ correctly represents truth in $M$ by Definition $4.92 \mathrm{~b}$ and 2c. So $\phi$ fails on all subsets of $M$ with cardinality $<\lambda$ by Definition 4.92 d. Thus 2 ) implies 1 ). Clearly if 1 ) holds we can construct a model $M$ satisfying 2 ).

We continue to use the conventions regarding $P^{M}, P_{1}^{M}$ from the proof of Lemma 4.10.

Definition 4.11 For $\psi$ as in Definition 4.10, $\operatorname{spec}\left(\psi, \theta, \kappa, A_{\tau, \phi}\right)$ is the set of the cardinalities of models $M$ of $\psi$ with $\left(P^{M}, P_{1}^{M}, Q, R_{1}^{M}\right) \approx\left(\theta, A_{\tau, \phi},<\right)$.

Theorem 4.12 For any cardinal $\theta$, the following four cardinals are equal.

1. $\lambda_{1}$ is the Hanf number of $P_{N}^{\theta}$.

2. $\lambda_{2}$ is the Löwenheim number of $L_{\theta^{+}, \omega}(I I)=\ell^{2}\left(L_{\theta^{+}, \kappa}(I I)\right)$.

3. $\lambda_{3}$ is the Löwenheim number of $L_{\theta^{+}, \theta^{+}}(I I)=\ell^{2}\left(L_{\theta^{+}, \theta^{+}}(I I)\right)$.

4. $\lambda_{4}=\sup \left\{\operatorname{spec}\left(\psi, \theta, \kappa, A_{\tau, \phi}\right): \psi \in L\left(I I, \tau_{*}\right), \phi \in L_{\theta^{+}, \theta^{+}}(I I)\right.$, and $A_{\tau, \phi} \subset \theta$ such that $\operatorname{spec}\left(\psi, \theta, \kappa, A_{\tau, \phi}\right)$ is bounded $\}$. 
Proof. We chose the logic $L_{\theta^{+}, \omega}$ precisely so $\lambda_{1} \leq \lambda_{2}$ (by a proof like that of Lemma 2.6 but now we have conjunctions of cardinality $\theta$ ). So in fact the demand that 'a model omits the type $p$ ' becomes 'the model satisfies the sentence in $L_{\theta^{+}, \omega}$, $\neg(\exists x) \wedge p$ ' so the worries in the first sections about the second order definability of $p$ disappear. Clearly $\lambda_{2} \leq \lambda_{3}$.

With $\psi$ from Lemma 4.10 and applying that lemma with $\tau$ as $\{=\}$ and with $\kappa=\theta^{+}$) yields:

$\left\{\min \left(\operatorname{spec}^{2}(\phi)\right): \phi \in L_{\theta^{+}, \theta^{+}}\right\} \subseteq\left\{\sup \left(\operatorname{spec}^{2}\left(\theta, \psi, A_{\tau, \phi}\right)\right): \phi \in L_{\theta^{+}, \theta^{+}}\right.$is bounded $\}$.

(We can replace $\phi$ by a $\phi^{*}$ whose only model is the model of $\phi$ with minimum cardinality to guarantee the containment.) Thus, $\lambda_{3} \leq \lambda_{4}$.

The proof that $\lambda_{4} \leq \lambda_{1}$ is obtained by modifying the proof of Theorem 2.5. Add to the vocabulary in the $T_{\phi}$ from the proof in section 3 of Theorem 2.5, symbols $P, P_{1}, Q, R_{1}$ and symbols $c_{\alpha}$ for each $\alpha \in A_{\tau, \phi}$ and use the same coding ideas to guarantee that $P_{1}, Q$ are contained in $P$ and all three are well-ordered by $R_{1}$. Thus, for $\phi \in L_{\theta^{+}, \theta^{+}}(I I)$ we can construct $\boldsymbol{t}_{\phi}$, encoding the second order sentence $\psi \in L\left(I I, \tau_{*}\right)$ defined in Definition 4.9 and where the type $p$ also codes that $Q^{M} \approx A_{\tau, \phi}$ so that the two spectra are related as in Theorem 2.5. The type $p$ is just $\{Q(x)\} \cup\left\{x \neq c_{\alpha}: \alpha \in A_{\tau, \phi}\right\}$. This yields $\lambda_{4} \leq \lambda_{1}$ by slightly modifying the argument for Lemma 2.9.

Our discussion of the Hanf and Löwenheim numbers of second order logic focused on two vocabularies: $\{=\}$ and $\tau_{*}$. In contrast, in many studies of the Hanf and Löwenheim numbers of logics the number is taken as the supremum for a given logic over all vocabularies of a bounded cardinality. That is, a Löweheim or Hanf function (with argument the cardinality of the vocabulary) is defined:

Definition 4.13 For any logic $\mathcal{L}$ and any cardinal $\theta$.

1. Let the Löwenheim function $\ell^{3}(\mathcal{L}, \theta)$ be the least cardinal $\mu$ such that for any vocabulary $\tau$ of cardinality $\leq \theta$ and any $\phi \in L_{\theta^{+},{ }_{\kappa}}(I I)(\tau)$, if $\phi$ has a model it has one of cardinality less than or equal $\mu$.

2. Let the Löwenheim number $h^{3}(\mathcal{L}, \theta)$ be the least cardinal $\mu$ such that for any vocabulary $\tau$ of cardinality $\leq \theta$ and any $\phi \in L_{\theta^{+},{ }_{\kappa}}(I I)(\tau)$, if $\phi$ has a model of cardinality $\mu$, it has arbitrarily large models.

The cardinalities of the vocabularies play a significant role. A trivial example is that both the Löwenheim number and Hanf number of first order logic is exactly the cardinality of the vocabulary. A more interesting example is that for the logic $L(Q)$ (with $Q$ interpreted as 'there exists uncountably many)', the Löwenheim number in $\tau=\{=\}$ is $\aleph_{1}$, while for an arbitrary vocabulary $\tau$ it is $\aleph_{1}+|\tau|$. The Hanf number of $L(Q)$ for countable vocabularies is $\beth_{\omega}$ (See e.g. 3.3.12 of [Sch85]). Thus,

$$
\beth_{\omega}=h^{3}\left(L_{\omega, \omega}(Q), \aleph_{0}\right)<h^{3}\left(L_{\omega_{1}, \omega}, \aleph_{0}\right)=\beth_{\omega_{1}} .
$$


Computing the Hanf number of $L(Q)$ for vocabularies of cardinality $\mu \geq \aleph_{1}$ is considerably more complicated. The key point is the following observation which does not depend on Assumption 0.3. Since we have not been able to find it in print we describe the key innovation here.

Theorem 4.14 If $\kappa$ is uncountable, $h^{3}\left(L_{\omega, \omega}(Q), \kappa\right)=h^{3}\left(L_{\omega_{1}, \omega}, \kappa\right)$.

Proof. Lopez-Escobar and Chang (e.g. [Cha68] showed how to code sentences of $L_{\lambda^{+}, \omega}$ as first order theories omitting types. And it is well-known (e.g. Theorem 5.4 of [She78]) that this can be reduced to omitting one type $p$ of the form $P(x) \cup\left\{x \neq c_{n}\right.$ : $n<\omega\}$. This translation can be done for $\tau$ of any cardinality. The crux of the current extension is that with an uncountable language one can further reduce the omission of the type $p$ to the assertion $\neg(Q x) P(x)$. For this, add to the language unary function symbols $F_{\alpha}$ for $\alpha<\aleph_{1}$ and a binary relation symbol $<$. Fix a family $\left\langle\eta_{\alpha}: \alpha<\omega\right\rangle$ of distinct functions from $\omega$ to 2 . Now add axioms asserting

1. $<$ linear orders $P$;

2. $c_{n+1}$ is the $<$-successor of $c_{n}$ and $c_{0}$ is $<-$ minimal;

3. Each $F_{\alpha}$ maps $P$ onto $P$;

4. For $\alpha<\beta<\aleph_{1}$, if $\left(\eta_{\alpha}\left\lceil n \neq \eta_{\beta}\lceil n)\right.\right.$ then $(\forall x)\left(c_{n}<x \rightarrow F_{\alpha}(x) \neq F_{\beta}(x)\right)$.

Now in any model of these axioms if the type $p$ is realized by some $c$, the $F_{\alpha}(c)$ for $\alpha<\aleph_{1}$ are uncountably many elements of $P$. This establishes the reduction. $\quad \square_{4.14}$

Remark 4.15 This result can extended substantially; we could have replaced the study of sentences of $L_{\omega_{1}, \omega}$ by theories of cardinality $\aleph_{1}$ without loss. Basic facts concerning the Hanf number of $\mathbb{L}_{\mu^{+}, \aleph_{0}}$ for vocabularies of cardinality $\mu \geq \aleph_{1}$ appear in VII.5 of [She78] and Chapter 4 of [Bal09]. General sufficient conditions to show $h^{3}\left(L_{\omega_{1}, \omega}, \kappa\right)=h^{3}\left(L_{\kappa^{+}, \omega}, \kappa\right)$ are in [GS05].

However, the dependence on the size of the vocabulary disappears for the Löwenheim number of infinitary second order logic. Without loss of generality we restrict to relational languages. There is no loss in our restriction to vocabularies of cardinality at most $\theta$ since no sentence of $L_{\theta^{+}, \kappa}(I I)$ can contain more than $\theta$ relation symbols.

Without loss of generality we restrict to relational languages. There is no loss in our restriction to vocabularies of cardinality at most $\theta$ since no sentence of $L_{\theta^{+}, \kappa}(I I)$ can contain more than $\theta$ relation symbols.

Note that just by existentially quantifying out the relation symbols, for any $\kappa \leq \theta^{+}$:

$$
\ell^{3}\left(L_{\theta^{+}, \kappa}(I I), \theta\right) \leq \ell^{2}\left(L_{\theta^{+}, \theta^{+}}(I I)\right) .
$$

Combining this observation with $\lambda_{2}=\lambda_{3}$ in Theorem 4.12, we have:

Corollary $4.16 \ell^{3}\left(L_{\theta^{+}, \kappa}(I I), \theta\right)=\ell^{2}\left(L_{\theta^{+}, \kappa}(I I)\right)$. 
We arrived at this result using our analysis of the Hanf number of $P_{N}^{\theta}$. But a variant of Corollary 4.16 can be obtained, using the same ideas of coding syntax, without the detour through $P_{N}^{\theta}$. It shows that with only a finite vocabulary one can code any reasonable similarity type.

Notation $4.17 \tau_{* *}$ is the vocabulary containing one unary predicate $P$, one binary relation symbol < and a ternary predicate $R$.

Theorem 4.18 $\ell^{3}\left(L_{\theta^{+}, \kappa}(I I), \theta\right)=\ell^{2}\left(L_{\theta^{+}, \kappa}(I I)\left(\tau_{* *}\right)\right)$.

Proof. Instead of considering an arbitrary vocabulary of size $\theta$ we can consider a 'universal' vocabulary $\sigma$ of $\theta$ binary relations. (It is easy to code the first in the second.) So the claim is that for any sentence $\phi \in L_{\theta^{+}, \kappa}(I I)(\sigma)$ there is a sentence $\phi^{*}$ of $L_{\theta^{+}, \kappa}(I I)\left(\tau_{* *}\right)$ such that $\operatorname{spec}(\phi)=\operatorname{spec}\left(\phi^{*}\right)$. (The converse is obvious.)

Let $\phi^{*}$ assert

1. $(P,<)$ is a well-order.

2. the formula obtained by replacing each occurence of $R_{i}\left(\sigma_{1}, \sigma_{2}\right)$ in $\phi$ by the formula (where $z$ does not occur in $\phi$ ):

$$
(\exists z)\left[P(z) \wedge \operatorname{otp}(\{y: P(y) \wedge y<z\})=i \wedge R\left(z, \sigma_{1}, \sigma_{2}\right)\right] .
$$

(Here $\sigma_{1}, \sigma_{2}$ are arbitrary terms from $\phi \in L_{\theta+, \kappa}(I I)(\sigma)$, i.e. individual variables.)

Now there is a model $N$ with cardinality $\lambda \geq \theta$ of $\phi^{*}$ where $P^{N}$ has order type $\theta$, such that $N \models \phi^{*}$ if and only if there is a $\sigma$-structure $M$ with cardinality $\lambda$ satisfying $\phi$.

\section{References}

[Bal09] John T. Baldwin. Categoricity. Number 51 in University Lecture Notes. American Mathematical Society, 2009. www.math.uic.edu/ jbaldwin.

[Cha68] C. C. Chang. Some remarks on the model theory of infinitary languages. In J. Barwise, editor, The syntax and semantics of infinitary languages, pages 36-64. Springer-Verlag, 1968. LNM 72.

[GS05] R. Goebel and S. Shelah. How rigid are reduced products? J Pure and Applied Algebra, 202:230-258, 2005.

[Han60] William Hanf. Models of languages with infinitely long expressions. In Abstracts of Contributed papers from the First Logic, Methodology and Philosopy of Science Congress, Vol.1, page 24. Stanford University, 1960.

[Mag71] M. Magidor. On the role of supercompact and extendible cardinals in logic. Israel Journal of Mathematics, 10:147-157, 1971. 
[New] Ludomir Newelski. Bounded orbits and measures on a group. preprint.

[Sch85] J. Schmerl. Transfer theorems and their applications to logics. In J. Barwise and S. Feferman, editors, Model-Theoretic Logics, pages 177-209. SpringerVerlag, 1985.

[She78] S. Shelah. Classification Theory and the Number of Nonisomorphic Models. North-Holland, 1978.

[Vaa79] Jouko Vaananen. On the Hanf numbers of unbounded logics. In B.Mayoh F.Jensen and K.Moller, editors, Proceedings from 5th Scandinavian Logic Symposium, pages 309-328. Aalborg University Press, 1979.

[Vaa82] Jouko Vaananen. Abstract logic and set theory II: Large cardinals. Journal of Symbolic Logic, 47:335-346, 1982. 